\title{
Mathematical Modelling and Simulation of the Thermal Processing of Anisotropic and Non-homogeneous Conduction- heated Canned Foods: Application to Canned Tuna
}

\author{
J. R. Banga,* A. A. Alonso, J. M. Gallardo \& R. I. Perez-Martin \\ Instituto de Investigaciones Marinas (CSIC), Eduardo Cabello 6, 36208 Vigo, Spain \\ *Present address: Department of Chemical Engineering, Facultad de Ciencias, \\ Universidad de Vigo, Apto. 874, 36200 Vigo, Spain.
}

\begin{abstract}
Thermal processing of conduction-heated canned foods is modelled using three approaches: (A) homogeneous isotropic, (B) homogeneous anisotropic and (C) nonhomogeneous anisotropic systems. Based on these models, numerical simulations are performed, using finite differences and finite elements methods. The simulation results are validated with experimental data obtained from the sterilization of canned tuna, a rather complex system. The last approach is found to be the most appropriate, though the second can be used for practical purposes.
\end{abstract}

\section{NOTATION}

$\mathrm{C}_{\mathrm{p}}$ Specific heat $\left(\mathrm{J} / \mathrm{kg}^{\circ} \mathrm{C}\right)$

F Single point integrated lethality ( $\mathrm{min}$ )

$\mathrm{F}_{\mathrm{c}}$ Critical point integrated lethality (min)

$\mathrm{h}$ Effective overall heat transfer coefficient $\left(\mathrm{W} / \mathrm{m}^{2}{ }^{\circ} \mathrm{C}\right)$

$\mathrm{k}$ Thermal conductivity $\left(\mathrm{W} / \mathrm{m}^{\circ} \mathrm{C}\right)$

$\mathrm{k}_{\mathrm{r}}$ Thermal conductivity in the radial direction $\left(\mathrm{W} / \mathrm{m}{ }^{\circ} \mathrm{C}\right)$

$\mathrm{k}_{\mathrm{z}}$ Thermal conductivity in the axial direction $\left(\mathrm{W} / \mathrm{m}{ }^{\circ} \mathrm{C}\right)$

L Half-height of a finite cylinder (m)

n Unit outward normal vector

$r$ Radial coordinate in the cylindrical system $(\mathrm{cm})$

$\mathrm{R}$ Total radius of a finite element $(\mathrm{cm})$

SSD Sum of squares of the differences 
SDR Standard deviation of the residuals

t Time (min)

T Temperature $\left({ }^{\circ} \mathrm{C}\right)$

$\mathrm{T}_{\mathrm{o}}$ Initial temperature of the canned product $\left({ }^{\circ} \mathrm{C}\right)$

$\mathrm{T}_{\mathrm{CR}}$ Critical point temperature $\left({ }^{\circ} \mathrm{C}\right)$

$\mathrm{T}_{\text {RET }}$ Retort temperature $\left({ }^{\circ} \mathrm{C}\right)$

$\mathrm{T}_{\text {sup }}$ Surface temperature $\left({ }^{\circ} \mathrm{C}\right)$

$\mathrm{X}_{\mathrm{T}}$ Total process time ( $\left.\mathrm{min}\right)$

z Axial coordinate in the cylindrical system $(\mathrm{cm})$

$\mathrm{Z}$ Temperature increment necessary for a tenfold reduction in $\mathrm{D}\left({ }^{\circ} \mathrm{C}\right)$

$\alpha$ Thermal diffusivity coefficient $\left(\mathrm{m}^{2} / \mathrm{s}\right)$

$\rho$ Density $\left(\mathrm{kg} / \mathrm{m}^{3}\right)$

\section{INTRODUCTION}

Thermal processing of canned foods is one of the food industry's operations that has received most attention in the scientific literature, which reflects the great economic and nutritional importance of this type of food processing. The development of mathematical models which permit a reliable simulation of this process is fundamental for its optimization (Teixeira et al., 1975; Saguy \& Karel, 1979; Nadkami \& Hatton, 1985; Banga et al., 1991a) and control (Teixeira \& Manson, 1982; Datta et al., 1986; Simpson et al., in press).

Numerous works exist on numerical simulation of thermal processing of conductionheated canned foods, based, in most cases, on the pioneering works of Teixeira et al. (1969a,b). An up-to-date review of these has been given by Banga (1991). For the most part, the models proposed in these works have been experimentally contrasted using only homogeneous foods (purees, pates, etc.) and/or homogeneous model systems (gels of bentonite, celite, carboxymethylcellulose, etc.).

Lu et al. (1991) have emphasized the necessity to produce models which allow the simulation of non-uniform external heating cases (heterogeneous containers and/or significant head spaces). On the other hand, to the authors' knowledge, no work considers the anisotropy of fibrous structured foods where the heat flow depends on its 
spatial direction, and in those which, in general, thermal conductivity is greater in the direction parallel to the fibres than in the perpendicular direction (Perez- Martin et al., 1989b).

For these reasons, one of the aims of this work was to develop a group of mathematical models which allow the consideration of situations of differing complexities in such a way that, by selection of the most suitable model, it is possible to simulate, with reasonable precision, the various forms of conduction-heated canned foods produced by the food industry. The simplest situation would be a homogeneous and isotropic system, and the most complicated would be a non-homogeneous and anisotropic one, with an intermediate anisotropic homogeneous system.

Another aim of this work was to select a fast and precise numerical method which permits an efficient solution of the formulated models. Finally, it is important to carry out an experimental validation of the results obtained by simulation, to demonstrate the usefulness of the models which consider anisotropy. To this end, we have carried out a series of experiments with white tuna (albacore) canned in oil. This food was chosen because of its economic importance and as a representative case of a non-homogeneous system (there is a layer of oil in the upper part of the canned product) and of an anisotropic one, as the thermal conductivities in the directions parallel and perpendicular to the muscle fibre differ by more than 15\% (Perez-Martin et al., 1989b).

\section{MATHEMATICAL MODELS}

Given that the construction of a model is an iterative process (Clark, 1978), and with the aim of embracing the different degrees of complexity which may occur, three mathematical models are subsequently shown which we will call $\mathrm{A}, \mathrm{B}$ and $\mathrm{C}$, corresponding to the three types of situations mentioned above. Cylindrical geometry will be considered in all cases as it is the most frequent. The extension of these models to other geometries does not present any conceptual problems.

Model A: homogeneous and isotropic system 
This is the simplest possible situation, and that traditionally covered in previous studies. The governing equation (Fourier's equation) for a finite cylinder of radius $\mathrm{R}$ and halfheight $\mathrm{L}$, for a homogeneous and isotropic medium, is:

$\frac{1}{\alpha} \frac{\partial T}{\partial t}=\frac{\partial^{2} T}{\partial r^{2}}+\frac{1}{r} \frac{\partial T}{\partial r}+\frac{\partial^{2} T}{\partial z^{2}}$

The initial condition is

$T=T_{0}\left\{\begin{array}{c}t=0 \\ 0 \leq r \leq R \\ -L \leq z \leq+L\end{array}\right.$

The boundary condition, where a profile of retort temperature depending on time, $\mathrm{T}_{\mathrm{RET}}(\mathrm{t})$, is considered, is

$$
T=T_{R E T}(t)\left\{\begin{array}{c}
0<t \leq X_{T} \\
r=R \\
z= \pm L
\end{array}\right.
$$

The symmetry conditions are

$$
\begin{aligned}
& \frac{\partial T}{\partial r}=0\left\{\begin{array}{c}
r=0 \\
0 \leq t \leq X_{T}
\end{array}\right. \\
& \frac{\partial T}{\partial z}=0\left\{\begin{array}{c}
z=0 \\
0 \leq t \leq X_{T}
\end{array}\right.
\end{aligned}
$$

Model B: homogeneous and anisotropic system

As an example of this type of product, we consider those canned anisotropic foods where head space or packing liquid upper layer thickness are of little significance. The governing equation for a finite cylinder is

$$
\frac{1}{\alpha_{r}} \frac{\partial T}{\partial t}=\frac{\partial^{2} T}{\partial r^{2}}+\frac{1}{r} \frac{\partial T}{\partial r}+\frac{K_{z}}{K_{r}} \frac{\partial^{2} T}{\partial z^{2}}
$$

with $\alpha_{\mathrm{r}}=\mathrm{k}_{\mathrm{r}} / \rho \mathrm{C}_{\mathrm{p}}$, and the same initial, perimeter and symmetry conditions as in Model A. It is evident that Model A is a particular case of B when $k_{z}=k_{r}=k$. Thus, a formulation for anisotropic materials would allow by default the consideration of isotropy. 
Model C: non-homogeneous and anisotropic system

This model permits the consideration of anisotropic products which also have head spaces and/or packing liquid layers of significant thickness. The effect of these on heat transmission will be modelled by the incorporation of a convective boundary condition in the upper part of the can, considering a single effective overall heat transfer coefficient.

The governing equation is the same as in Model B, eqn (6). The initial condition also coincides with eqn (2). With regard to the boundary conditions, if the thickness of the packing liquid considered is $\mathrm{L}_{\mathrm{c}}=\mathrm{L}-\mathrm{L}_{\mathrm{F}}$ (see Fig. 1), eqn (3) is replaced by

$$
\begin{aligned}
& T=T_{R E T}(t)\left\{\begin{array}{c}
0<t \leq X_{T} \\
r=R \\
z=-L
\end{array}\right. \\
& k \cdot \frac{\partial T}{\partial n}=h \cdot\left[T_{S U P}-T_{R E T}(t)\right]\left\{\begin{array}{c}
0<t \leq X_{T} \\
z=+L_{F}
\end{array}\right.
\end{aligned}
$$

where $\mathrm{h}$ is the effective overall heat transfer coefficient. With respect to the symmetry conditions, only that of radial symmetry (eqn (4)) will be valid, because the boundary condition (8) breaks the axial symmetry of the system, considered in the other models by eqn (5).

The critical point integrated lethality, $F_{c}$, which is the customary form used in the food industry, will be calculated in the same way for the three models:

$$
F_{c}=\int_{0}^{X_{T}} 10^{\left[T_{C R}(t)-T_{R E F}\right] / Z}
$$

where $T_{C R}(t)$ is the thermal history of the said point. In the classical formulation (Model A) it can be assumed that this point coincides with the geometric centre, the error of this assumption being small (Hicks, 1951; Teixeira et al., 1969b; Flambert \& Deltour, 1972). However, as will be shown below, in Models B and C, not only will it not coincide with the geometric centre but it will also be a non-stationary point, the position of which evolves along the central axis, and particularly rapidly at the beginning of the cooling stage. 


\section{NUMERICAL METHODS}

The simulation of a process necessitates a mathematical model and an algorithm which permits its numerical solution for certain conditions. The previously shown models belong to those denominated distributed parameter systems, where the governing equations are of the partial differential type. Basically, there are two types of numerical techniques for this problem: the finite differences (FD) and the finite element (FE) methods.

One of the main differentiating characteristics of FD and FE methods is that computer programs for the latter are frequently of a general character, valid for any geometry, whereas those of the FD method are specific for each system. Thus, although it is true that the FE method programming effort is greater than that of the FD method for a given system, in the FD method it is necessary to write a new program each time variations are introduced in the geometry or boundary conditions.

The main disadvantage of the FE method is the enormous computation time required, as compared with the FD method. Consequently, when geometry and boundary conditions are not complicated, FD methods are preferable (Jaluria \& Torrance, 1986).

In this work, FD methods have been used for the solution of Models A and B, whereas for Model $\mathrm{C}$ an FE method has been chosen because of its great flexibility for changing boundary conditions.

Finite differences methods

Computer programs have been written (using FORTRAN 77) based on the FD approximations for Models A and B according to the explicit FTCS method (forward time central space; Arpaci, 1966), the ADEP method (alternate direction explicit procedure; Allada \& Quon, 1966), the ADIP method (alternate direction implicit procedure; Douglas, 1955), and also the new MVG method (modified variable grid; Simpson et al., in press). 
Based on a comparative study of the ratio between precision and computing time (Banga, 1991), the FTCS and MVG methods were chosen as the most suitable. The explicit FTCS method was used by Teixeira et al. (1969a,b) for the first time and, since then, several slight modifications have been published.

The MVG method has been successfully used for the control (Simpson et al., in press) and multipurpose optimization (Banga et al., 1991b) of the process, and is based on an ADEP applied to a variable step grid. The nodal coordinates of this grid correspond to the roots of Legendre's polynomials of order equal to the number of nodes. This allows the evaluation of spatial integrals (overall nutrient retention, overall integrated lethality, energy absorbed by the product, etc.) by Gaussian quadrature, which provides a high accuracy and efficiency.

The FTCS and MVG methods are indicated in particular for those simulations where it is necessary to sample the heating temperature at short time intervals, in which case an implicit procedure would be far more costly from a computational point of view. Typical examples of this situation are variable retort temperature profiles and on-line process control.

Finite elements method

In this investigation, an FE package from the Department of Applied Mathematics of the University of Santiago de Compostela (Bermudez \& Martinez, 1990) was used. Although, initially, trials were carried out with a more general 3D version, the enormous computing time and memory consumed made it necessary to select a 2D version, because in the geometry considered (finite cylinder) a symmetry axis exists.

The package consists of a set of FORTRAN 77 subroutines which permit, in a first step, the generation of a 2D grid corresponding to a determined system and later, the calculation of nodal thermal histories, once the initial and boundary conditions have been fixed. To fix a determined boundary condition, it is sufficient to indicate its code and type (Dirichlet or convective), including the effective heat transfer coefficient value if it is of the convective type. 
The thermal history calculation subroutines use Garlekin's method in space, applied to an FE grid of triangular order one (P1) elements, together with a totally implicit (CrankNicholson) FD method in time. The original set of subroutines was completed with others to calculate:

single point lethalities in a specific set of nodes; localization of the coldest point during the process; localization of the least lethality (critical) point during the process; overall and single point nutrient retentions.

Thus, the resultant package, called EF2D, permits the simulation of the effect of nonsymmetrical boundary conditions on the localization of the coldest and critical points. To our knowledge, only Naveh et al. (1984) have simulated some cases related to this situation.

\section{EXPERIMENTAL}

Tuna canning

Fresh white tuna (albacore, Thunnus alalunga), was acquired at a local market and stored at $-40^{\circ} \mathrm{C}$ until processing. The fish were defrosted, headed, gutted and steam precooked, according to the method of Perez-Martin et al. (1989a). Once cooked, white muscle was separated from the skin, bones and dark muscle.

Two types of cylindrical commercial containers were used: $\mathrm{RO}-100(\mathrm{R}=3 \cdot 26 \mathrm{~cm}, \mathrm{~L}=15$ $\mathrm{cm})$ and $\mathrm{RO}-1150(\mathrm{R}=7 \cdot 5 \mathrm{~cm}, \mathrm{~L}=3 \cdot 5 \mathrm{~cm})$ cans. The filling of these cans with tuna white muscle was done automatically in the case of the RO-100, and manually in the case of RO-1150, because of its large size. The meat was always cut across the grain.

Afterwards, neutralized olive oil was added, and the cans were hermetically sealed.

Thermal treatments: heat penetration data acquisition

Thermal treatments were carried out in a 125-litre horizontal steam retort, fed by a 350 $\mathrm{kg} / \mathrm{h}$ steam generator and connected to an air compressor. The retort operation was done 
in three stages: venting (3-4 min), heating at constant temperature and cooling with pressurized water.

Heat penetration data and retort temperature were measured and registered using type $\mathrm{T}$ thermocouples (O. F. Ecklund, FL, USA) connected to an HP-3412A data acquisition system (Hewlett Packard, CA, USA), which was linked to a PC-AT computer using a GPIB-PC IIA-1 interface card (National Instruments, TX, USA). A computer program, FCCAL, was used for the on-line monitoring of temperatures and F, values of up to six thermocouples. FCCAL allows these values to be presented on the screen numerically and graphically simultaneously, and at the same time stored on disk in an ASCII file for later analysis and manipulation. This program was developed using the libraries of the Lab Windows software package (National Instruments, TX, USA).

Thermal diffusivity calculation

To be able to carry out numerical simulations of the process, thermal diffusivity needs to be determined from experimental heat penetration data. Numerous methods can be found in literature for this purpose. Excellent revisions have been made by Singh (1982), Perez-Martin (1986) and Murakami and Okos (1988). In this study, two methods have been used to calculate thermal diffusivity from the time-temperature history of the geometrical centre of the cans:

(1) The $f_{h}$ method (classical): this method has been described by Ball and Olson (1957). The $f_{h}$ parameter is obtained by linear regression of the straight part of the semi-logarithmic heating curve. Thermal diffusivity is then calculated from $\mathrm{f}_{\mathrm{h}}$ by a simple formula. This method assumes isotropy and constant heating temperature.

(2) The least-squares method: many variations of this method have been described. Basically, it consists in obtaining thermal diffusivity by minimization of the squares of the differences between the experimental temperature and that calculated by the numerical or analytical solution of Fourier's equation.

In this study, we have developed a computer program, MINT, based on the minimization of the sum of squares of the differences (SSD) concept, where the calculated temperature is obtained by the numerical solution of the model selected by 
the user to represent the system considering the real retort temperature profile $T_{\text {RET }}(t)$. On the other hand, MINT allows the evaluation of the SSD in a time interval chosen by the user, which permits the elimination of parts of the thermal history that could otherwise bias the thermal diffusivity final value. The algorithm used for the minimization is Powell's univariate search procedure (routine ZXLSF of the IMSL library).

To measure goodness of fit, the program gives the standard deviation of the residuals (SDR) and the final SSD. From these parameters, it is possible to compare statistically the adjustment of the three models for a determined experimental system, and therefore be able to rigorously select the best.

Effective heat transfer coefficient calculation

Model C permits the simulation of simple non-homogeneous systems by considering a non-infinite effective overall heat transfer coefficient in the upper (or other) surface boundary. This implies that an experimental estimation of this coefficient is needed before simulation with this model.

In this work, we have made a simultaneous calculation of thermal diffusivity and the effective heat transfer coefficient based again on the least-squares concept, where the SSD between experimental and predicted (by Model C) temperatures is minimized. For this, a second version of the MINT program described above was used, with Powell's univariate search procedure being replaced by the ICRS (integrated controlled random search) multi-variate algorithm (Banga \& Casares, 1987).

To increase the number of degrees of freedom, thermal histories of several points (i.e. distributed along the radius or the symmetry axis) may be considered. The experimental measurement of these profiles implied the placing of several thermocouples inside each can. To minimize experimental errors created by possible conduction effects (Cowell et al., 1959), it was thought preferable to carry out these experiments in the largest format available (RO-1150).

\section{RESULTS AND DISCUSSION}


In the first place, we considered the case of canned tuna in RO-100 cans where the thickness of the packing liquid layer was not very significant (about 2-3 mm), with a measured drained weight per can of $83 \pm 3 \mathrm{~g}$ (the legal minimum is $67 \mathrm{~g}$ ). To evaluate the experimental variability between experimental heat penetration curve replicates, and thus to be able to judge on a statistical basis the precision of the simulation results, the SDR was calculated following Lenz and Lund (1977). The experiments with the above cans gave a result of $0.4+0.3^{\circ} \mathrm{C}$ for SDR.

The thermal diffusivity value determined using the classic $\left(f_{h}\right)$ method for a total of six processes was $\alpha=1.11 \times 10^{-7} \pm 0.02 \times 10^{-7} \mathrm{~m}^{2} / \mathrm{s}$. Figure 2 shows the semi-logarithmic plot of the dimensionless centre temperature against time for one of these experiments. The linear correlation coefficients $\left(r^{2}\right)$ for the $f_{h}$ calculation varied between 0.996 and 0.999. This thermal diffusivity value, even if it is not to be used as a simulation parameter, is a good initial value for the MINT program, which calculates the thermal diffusivity more rigorously, taking into account both the model chosen by the user and the real retort temperature profile.

The thermal diffusivity value for Model A, calculated using the MINT program, was $\alpha$ $=1.26 \times 10^{-7} \pm 0.03 \times 10^{-7} \mathrm{~m}^{2} / \mathrm{s}$, with a final result for the SDR of $1.0 \pm 0.4^{\circ} \mathrm{C}$. It must be emphasized that, using the thermal diffusivity obtained with the $f_{h}$ method, the SDR for Model A was $2.5 \pm 0.4^{\circ} \mathrm{C}$. Therefore, the use of the MINT program is clearly preferable to the $\mathrm{f}_{\mathrm{h}}$ method.

When considering Model B (homogeneous and anisotropic system), with $\mathrm{k}_{\mathrm{z}} / \mathrm{k}_{\mathrm{r}}=1.154$, taken from Perez-Martin et al. (1989b), the MINT program gave, for the same six experiments, an $\mathrm{QT}=1.13 \times 10^{-7} \pm 0.03 \times 10^{-7} \mathrm{~m}^{2} / \mathrm{s}$. The corresponding SDR was $0.6 \pm$ $0.3{ }^{\circ} \mathrm{C}$. Using a t-test it was verified that the SDR of Model B was not significantly different from the experimental value $(\mathrm{P}<0-05)$, whereas that of Model A certainly was. It was therefore demonstrated that, for the specific case of canned tuna, the proposed Model B permits more reliable simulations than the traditional Model A. 
Figure 3 shows a typical plot of experimental centre point temperature against time, together with the results obtained by simulation using Model B. Very good agreement is observed between the two models. Also, the central point lethality values obtained by simulation with Model B agree very well with the lethal values determined experimentally for a total of six treatments, as can be seen in Fig. 4. It has been proven that Model B permits a sufficiently precise simulation of the experimental results in the case of canned tuna with a packing oil layer of small thickness (about $2 \mathrm{~mm}$ ).

However, and as was expected, experimental heat penetration curves obtained using cans with a significant thickness of packing oil layer (about $5 \mathrm{~mm}$, drained weight per can $72 \pm 2 \mathrm{~g}$ ) showed considerable discrepancies from simulation results using Model $\mathrm{B}$, especially at the beginning of the process. It was therefore decided to use Model C, in which the effect of the oil layer can be modelled using an overall effective heat transfer coefficient.

As the first step in using Model C, the corresponding thermophysical parameters (the overall effective heat transfer coefficient, $h$, and the thermal diffusivity, $\alpha$ ) were calculated from heat penetration data using the MINT program. For the former, a value of $\mathrm{h}=90 \pm 10 \mathrm{~W} / \mathrm{m}^{2}{ }^{\circ} \mathrm{C}$ was obtained, which is within the range reported by Naveh (1982) for various head spaces. For the latter, a value of $\alpha=1.29 \times 10^{-7} \pm 0.04 \times 10^{-7}$ $\mathrm{m}^{2} / \mathrm{s}$ was obtained, in agreement with results calculated by de1 Valle and Yeannes (1982) and Perez-Martin et al. (1989b) for cans with similar packing conditions.

The differences between this new $\alpha$ value and those determined in the previously described experiments occur because the can filling procedures were different, so that significant differences in the degree of tuna muscle packing resulted. It is concluded that the determination of the model parameters, and in particular that of the thermal diffusivity, must be repeated whenever the filling and packing conditions are varied.

Figure 5 shows a typical experimental thermal history of the geometric centre for an RO-100 can, together with those calculated using Models A, B and C, which clearly shows that Model $\mathrm{C}$ is better adjusted to the experimental reality, whereas for Models $\mathrm{A}$ and $\mathrm{B}$ there exist important differences. 
Figure 6 shows the experimental and calculated (by simulation) values of the central point integrated lethality $\left(\mathrm{F}_{\mathrm{c}}\right)$ against time for the same experiment. Both Models B and C give good lethality estimations, so that even in these cans where the insulating effect of the upper oil layer is important, Model B can be reliably used to calculate processing times.

Though Model C is more rigorous and, as has been shown, predicts the thermal histories better than model B, the computation time necessary is much higher, because FEs are used to solve Model C, whereas Model B is solved by the FD method. Therefore, from the practical point of view, Model B is preferable to Model C.

However, Model C implies a series of consequences which must be adequately evaluated: as a result of the asymmetry of the boundary conditions, the temperature distribution will be equally asymmetrical, and this means that the geometric centre of the container will not be the critical point. Furthermore, by simulation using Model $\mathrm{C}$ it can be proven that this critical point, considered as that of least lethality at each moment, varies its position during the process. Naveh et al. (1984) obtained a similar result in their study on the consequences of placing a can with an end flat against the retort bottom on the location of the coldest zone.

Figure 7 shows the isotherms, calculated by simulation using Model C, for an RO-100 can processed at $115^{\circ} \mathrm{C}$. The above-mentioned asymmetry is clearly observed. Figure 8 represents single point integrated lethality values, $\mathrm{F}$, for various points on the $\mathrm{z}$-axis of the RO-100 can before and after the cooling stage. It can be seen how the z-coordinate corresponding to the $\mathrm{F}$ minima has varied, although the final $\mathrm{F}$ difference between the geometric centre and that minima is negligible. This result is in agreement with an earlier and more qualitative study on the effect of head spaces made by Evans and Board (1954).

However, although this effect of movement of the critical point may be negligible in a small can such as the RO-100 considered here, it is reasonable to suppose that in a large can the final $\mathrm{F}$ differences between the geometric centre and the true critical point could be important. Given that the normal practice in industry is to consider the geometric centre as the critical point, and that the thermocouples are placed there for the 
experimental lethality measurement, we thought it necessary to investigate experimentally and by simulation the axial distribution of single point lethality in large cans, to evaluate the possible risks in such a practice.

For this purpose, three experiments with RO-1150 cans were carried out, in which four thermocouples were placed in each can at various heights along the z-axis. In all cases, one of the thermocouples was situated at the geometric centre. These cans were processed to different $\mathrm{F}_{\mathrm{c}}$ values, monitoring the thermal histories with the FCCAL program. These heat penetration data were then used by the MINT program to calculate the parameters of Model C. The results were $\alpha=1.64 \times 10^{-7} \pm 0.05 \times 10^{-7} \mathrm{~m}^{2} / \mathrm{s}$ and $\mathrm{h}=$ $48 \pm 10 \mathrm{~W} / \mathrm{m}^{2}{ }^{\circ} \mathrm{C}$.

The thermal diffusivity value, which was very high compared with those determined for the RO-100 cans, was rather surprising. The overall effective heat transfer coefficient was somewhat lower than those determined in RO-100 cans, which is logical considering that the thickness of the oil layer is greater.

Both the $\alpha$ and $h$ values show a great variability. There is a twofold explanation: on the one hand, the cans have been packed manually, and on the other, given the characteristics of the tuna muscle, it is virtually impossible in this size of can to avoid gaps which, during thermal processing, permit the appearance of convection currents in the packing oil. For this reason, the heat transfer mechanism is no longer purely conductive but becomes a mixture of conduction and convection. Given that Model C considers only conduction for the tuna muscle, the final effect is an effective thermal diffusivity greater than that previously determined for small cans where heat transfer was purely conductive.

Figure 9 gives the single point $\mathrm{F}$ values, determined experimentally and calculated by simulation with Model $\mathrm{C}$, along the z-axis for three processes. In all cases the agreement between observed and predicted values is good. For processes 1 and 2, the single point $\mathrm{F}$ varies little in the zone under consideration. However, for process 3 , which is that of greatest $T_{\text {RET }}$, a minimum $F$ value of 7.6 min exists, corresponding to a 
$\mathrm{z}=3.1 \mathrm{~cm}$ whereas the $\mathrm{F}$ of the geometric centre, $\mathrm{z}=3.5 \mathrm{~cm}$, is $8.8 \mathrm{~min}$. That is to say, the true critical point is found $4 \mathrm{~mm}$ below the geometric entre and has a $\mathrm{F}$ value $14 \%$ lower than that at the geometric centre.

Although this difference may be permitted in practice (taking into account that variability in other process parameters, such as can filling, may imply higher variabilities in the $\mathrm{F}_{\mathrm{c}}$ value), simulation with Model $\mathrm{C}$ gives a reliable evaluation of all these factors.

\section{ACKNOWLEDGEMENTS}

This work was financially supported by a research grant from the Comision Interministerial de Ciencia y Tecnologia (ALI88-0 145CO2-02, Spain).

\section{REFERENCES}

Allada, S. R. \& Quon, D. (1966). A stable, explicit numerical solution of the conduction equation for multidimensional nonhomogeneous media. Heat Transfer - L.A., 62(64), 151-6.

Arpaci, V. S. (1966). Conduction Heat Transfer. Addison-Wesley, Reading, MA, pp. 483-92.

Ball, C. O. \& Olson, F. C. W. (1957). Sterilization in Food Technology. McGraw-Hill, New York, pp. 193-200.

Banga, J. R. (1991). Simulación y optimización de1 procesamiento térmico de conservas de alimentos. PhD thesis, Department of Chemical Engineering, University of Santiago de Compostela.

Banga, J. R. \& Casares, J. J. (1987). ICRS: application to a wastewater treatment plant model. IChemE Symp. Ser., 100, 183-92.

Banga, J. R., Pérez-Martin, R. I., Gallardo, J. M. \& Casares, J. J. (1991a). Optimization of the thermal processing of conduction-heated canned foods: study of several objective functions. J. Food Eng., 14 (1), 25-51.

Banga, J. R., Simpson, R., Almonacid, S. \& Torres, J. A. (1991b). MOPT: multipurpose optimization of thermal processing. Presented at 52nd Annual Meeting of the Institute of Food Technologists (IFT91), 1-5 June, Dallas, TX. 
Bermudez, A. \& Martinez, A. (1990). Optimal control of industrial sterilization of canned foods. Proc. V: IFAC Symp. on Distributed Parameter Systems, Perpignan. Pergamon Press, Oxford, pp. 37-42.

Clark, J. P. (1978). Mathematical modeling in sterilization processes. Food. Technol., $32,73-5$.

Cowell, N. D., Evans, H. L., Hicks, E. W. \& Mellor, J. D. (1959). Conduction errors in thermocouples used for heat penetration measurements in foods which heat by conduction. Food Technol., 13 (8), 425-9.

Datta, A. K., Teixeira, A. A. \& Manson, J. E. (1986). Computer-based retort control logic for on-line correction of process deviations. .J. Food Sci., 51 (2), 480-3.

de1 Valle, C. \& Yeannes, M. I. (1982). Difusividad térmica efectiva de bonito (Sarda sarda) en aceite. Lat. Am. J. Heat Mass Transf. 6, 31-9.

Douglas, J. (1955). On the numerical integration of $(\mathrm{du} / \mathrm{dx})+(\mathrm{du} / \mathrm{dy})=\mathrm{du} / \mathrm{dt}$ by implicit methods. J. Sot. Indust. Appl. Math., 3, 42-65.

Evans, H. L. \& Board, P. W. (1954). Studies in carming processes I. Effect of headspace on heat penetration in products heating by conduction. Food Technol., 8, 258-64.

Flambert, F. \& Deltour, J. (1972). Localization of the critical area in thermally processed conduction heated canned food. Lebensm. - Wiss. u. Technol., 5 (1), 7 13.

Hicks, E. W. (1951). On the evaluation of carming processes. Food Technol., 5, 134-42. Jaluria, Y. \& Torrance, K. E. (1986). Computational Heat Transfer. Hemisphere, Washington, DC, pp. 81-142.

Lenz, M. K. \& Lund, D. B. (1977). The lethality-Fourier number method: experimental verification of a model for calculating average quality factor retention in conduction-heated, canned foods. J. Food Sci., 42, 989-1001.

Lu, Q., Mulvaney, S. J. \& Hsieh, F. (1991). Thermal processes for metal cans compared to retortable plastic containers. J. Food Sci., 56 (3), 835-7.

Murakami, E. G. \& Okos, M. R. (1988). Measurement and prediction of thermal properties of foods. NATO Advanced Research Workshop, 16-21 October, Porto (Portugal), pp. 1-37.

Nadkarni, M. M. \& Hatton, T. A. (1985). Optimal nutrient retention during the thermal processing of conduction-heated canned foods: application of the distributed minimum principle. J. Food Sci., 50, 1312-21. 
Naveh, D. (1982). Analysis of transient conduction heat transfer in the thermal processing of food using the finite element method. $\mathrm{PhD}$ thesis, University of Minnesota, St Paul, MN.

Naveh, D., Pflug, I. J. \& Kopelman, I. J. (1984). Sterilization of food in containers with an end flat against a retort bottom: numerical analysis and experimental measurements. J. Food Sci., 49, 461-7.

Pérez-Martín, R. I. (1986). Estudio de los procesos térmicos en la fabricación de conservas de atún blanco y su incidencia en la calidad. $\mathrm{PhD}$ thesis, Department of Chemical Engineering, University of Santiago de Compostela.

Pérez-Martín, R. I., Banga, J. R., Sotelo, M. C., Aubourg, S. \& Gallardo, J. M. (1989a). Prediction of precooking times for albacore (Thunnus alalunga) by computer simulation. J. Food Eng., 10, 83-95.

Pérez-Martín. R. I., Gallardo, J. M., Banga, J. R. \& Casares, J. J. (1989b).

Determination of thermal conductivity, specific heat and thermal diffusivity of albacore (Thunnus alalunga). Z. Lebensm. Unters. Forsch., 189, 525-9.

Saguy, I. \& Karel, M. (1979). Optimal retort temperature profile in optimizing thiamine retention in conduction-type heating of canned foods. J. Food Sci., 44, 1485-90.

Simpson, R., Almonacid, S. \& Torres, J. A. (in press). Mathematical models and logic for the computer control of batch retort process operations: conduction- heated foods. J. Food Eng.

Singh, R. P. (1982). Thermal diffusivity in food processing. Food Technol., 36, 87-91.

Teixeira, A. A. \& Manson, J. E. (1982). Computer control of batch retort operations with on-line correction of process deviations. Food Technol., 4, 85-90.

Teixeira, A. A., Dixon, J. R., Zaradnik, J. W. \& Zinsmeister, G. E. (1969a). Computer optimization of nutrient retention in the thermal processing of conduction-heated foods. Food Technol., 23, 137-42.

Teixeira, A. A., Dixon, J. R., Zaradnik, J. W. \& Zinsmeister, G. E. (1969b). Computer determination of spore survival distributions in thermally-processed conductionheated foods. Food Technol., 23, 78-80.

Teixeira, A. A., Zinsmeister, G. E. \& Zaradnik, J. W. (1975). Computer simulation of variable retort control and container geometry as a possible means of improving thiamine retention in thermally processed foods. J. Food Sci., 40, 656-9. 
Fig. 1. Geometrical characterization of a non-homogeneous conduction-heated canned food (Model C).

Fig. 2. Semi-log heating curve (dimensionless centre temperature vs time) for an RO100 can with negligible upper oil layer.

Fig.3. Temperature at centre of RO-100 tuna can vs time plot: comparison of experimental and simulation values. Retort temperature $\left(T_{\mathrm{RET}}\right)$ is also represented.

Fig. 4. Predicted (by simulation using Model B) vs measured critical point integrated lethality $\left(\mathrm{F}_{\mathrm{c}}\right)$ values.

Fig. 5. Experimental critical point temperature-time plot compared with simulation results using Models $\mathrm{A}, \mathrm{B}$ and $\mathrm{C}$.

Fig. 6. Experimental critical point integrated lethality vs time plot, compared with simulation results using Models A, B and C.

Fig. 7. Temperature isotherms for an RO-100 tuna can obtained by simulation using Model C (solved by the FE method).

Fig. 8. Single-point integrated lethality (F) profiles along the axis of an RO-100 can after the heating and cooling periods. Simulation made using Model C.

Fig. 9. Measured and simulated (using Model C) single-point integrated lethality profiles along the axis of an RO-1150 can for three processes. 